\title{
Rezension: \\ Dominik Elser, Die privatisierte Erfüllung staatlicher Aufgaben
}

Dominik Elser geht in seiner Dissertation der Frage nach, welche Probleme und Spielräume aus verfassungsrechtlicher Sicht auftreten, wenn staatliche Aufgaben privatisiert erfüllt werden. Die Untersuchung zeigt auf, dass Spielräumen in Bezug auf eine grössere Flexibilität, einzelfallgerechten Erfüllungsweisen und eine erhöhte Akzeptanz des staatlichen Handelns Probleme in Form von zusätzlichem Kontrollaufwand, Interessenkonflikten und einer erschwerten Erkennbarkeit des staatlichen Handelns gegenüberstehen.

\section{Einleitung}

II. Inhalt

Zitiervorschlag:

PHIL BAUMANN, Rezension: Dominik Elser, Die privatisierte Erfüllung staatlicher Aufgaben, sui generis 2020, S. 425 


\section{Einleitung}

1 Bisher standen bei der Bearbeitung der privatisierten Erfüllung staatlicher Aufgaben die Gründe für eine Auslagerung sowie deren verfassungsrechtliche Zulässigkeit im Vordergrund. ${ }^{1}$ In seiner Dissertation geht es Elser allerdings weder darum, die Zulässigkeit der Erfüllung staatlicher Aufgaben durch Private noch die Motive dafür auszuloten. Vielmehr untersucht die Arbeit die Frage, in welchem Verhältnis die privatisierte Erfüllung öffentlicher Aufgaben zu den verfassungsrechtlichen Anforderungen an das staatliche Handeln steht. Dabei werden vom Autor Problemfelder und Spielräume aufgezeigt, die infolge der verfassungsrechtlichen Anforderungen entstehen.

\section{Inhalt}

2 Voraussetzung für diese Analyse ist in einem ersten Schritt die begriffliche Klärung der "privatisierten Erfüllung staatlicher Aufgaben» (\$2). Stilsicher steuert hier Elser durch die Klippen der Begrifflichkeiten. Er definiert vorerst staatliche Aufgaben (S. $7 \mathrm{ff}$.) und wendet sich daraufhin deren Privatisierung zu (S. 16). Dabei werden in der Arbeit zwei Arten der Privatisierung unterschieden: Eine «Aufgabenprivatisierung» liegt vor, wenn der Staat auf die Erfüllung einer bisherigen staatlichen Aufgabe verzichtet und diese den Privaten überlässt. Demgegenüber bleiben bei der «Erfüllungsprivatisierung», die im Fokus der Dissertation steht, der Aufgabenbestand und die Aufgabenverantwortung des Staates unberührt. Die Aufgabe bleibt staatlich, «nur» die Art und Weise ihrer Erfüllung wird privatisiert (S. 23). Anschliessend ordnet Elser die Vielfalt der bestehenden Privatisierungsbegriffe (echte und unechte, materielle und formelle Privatisierung; Organisations-, Teil-, Finanzierungs-, und Vermögensprivatisierung) in diese Zweiteilung ein und nimmt hilfreiche Abgrenzungen zu weiteren im $\mathrm{Zu}$ sammenhang mit Privatisierungen verwendeten Begriffen (wie Dezentralisierung, Auslagerung, Deregulierung, Liberalisierung, New Public Management) vor (S. 25ff.). Vor dem Hintergrund dieser verwirrenden Fülle an Privatisierungsphänomenen und -begrifflichkeiten ist Elser zuzustimmen, dass der Begriff der Erfüllungsprivatisierung die Privatisierungsdiskussion vereinfachen kann.

1 Vgl. dazu etwa TOBIAS JAAG, Dezentralisierung und Privatisierung öffentlicher Aufgaben: Formen, Voraussetzungen und Rahmenbedingungen, in: Jaag (Hrsg.), Dezentralisierung und Privatisierung öffentlicher Aufgaben, Referate einer Tagung vom 29. September 1999, Zürich 2000, S. 23 ff.; BERNHARD RÜTSCHE, Staatliche Leistungsaufträge und Rechtsschutz, ZBJV 2016, S. 73 ff.
Allerdings bleibt mit Bezug auf die Erfüllungsprivatisie- 3 rung die Schwierigkeit, zu bestimmen, in welchen Fällen von einer privatisierten Erfüllung staatlicher Aufgaben auszugehen ist. Elser wählt hier einen äusserst weitgehenden Ansatz: Eine privatisierte Aufgabenerfüllung liegt demnach vor, wenn sie einer privaten Tätigkeit ähnelt und damit ebenso gut im privatautonomen, wettbewerbswirtschaftlichen Bereich angesiedelt werden könnte (S. 24f.). Die Art und Weise, wie die staatliche Aufgabe erfüllt wird, wirkt - von aussen her betrachtet - privat (S. 89). Diese Grenzziehung erlaubt dem Autor zwar, «die Gemeinsamkeiten von sehr unterschiedlichen Vorgängen zu untersuchen» (S.24f.). Trotzdem bleiben Zweifel, ob die «Ähnlichkeit» zu privaten Tätigkeiten ein sachgerechtes Abgrenzungskriterium darstellt. Mittlerweile werden zahlreiche Verwaltungstätigkeiten in einer Weise vorgenommen, die privater Tätigkeit ähnelt. Man denke bspw. an die «Online-Schalter» von Gemeinden, in denen wie in einem Online-Shop Heiratstermine reserviert und Bestätigungen sowie Auszüge (z.B. Wohnsitzbestätigungen) in den Warenkorb gelegt und bestellt werden können. Damit besteht die Gefahr, dass der Untersuchungsgegenstand der privatisierten Erfüllung staatlicher Aufgaben konturlos wird.

Elser begegnet dieser Gefahr in $\$ 4$ seiner Dissertation. 4 Zunächst legt er dar, dass eine Erfüllungsprivatisierung bei jedem Aspekt der Aufgabenerfüllung ansetzen kann (S. 90). Im Fokus der Untersuchung liegen indessen Erfüllungsaspekte, die für Private direkt in Erscheinung treten. Dabei handelt es sich um die Organisations- und Handlungsformen der Verwaltungsträger, sowie die Finanzierungsformen, mit denen die Erfüllung staatlicher Aufgaben bezahlt wird (S. 95). Anschliessend wird ausdifferenziert, was die Privat-Ähnlichkeit mit Blick auf die verschiedenen Organisations-, Handlungs- und Finanzierungsformen bedeutet. Dazu nimmt Elser jeweils in einem ersten Schritt eine sorgfältige Auslegeordnung der verschiedenen Organisations-, Handlungs- und Finanzierungsformen vor. In einem zweiten Schritt legt er dar, welche Formen als "privatisiert» betrachtet werden, wobei zurecht darauf hingewiesen wird, dass eine klare Grenzziehung unmöglich ist. Die Ähnlichkeit zu privaten Organisationen, privatem Handeln oder privaten Aufgabenfinanzierungen dienen Elser hierbei als Richtschnur. Schliesslich werden Kennzeichen der Erfüllungsprivatisierung in organisatorischer, instrumenteller und finanzieller Hinsicht herausgearbeitet, die bereits einen ersten Überblick über die Chancen und Risiken der verschiedenen Erfüllungsprivatisierungen geben. So zeigt der Autor beispielsweise auf, dass privatisierte Handlungsformen mehr Gestaltungsfreiheit im Einzelfall zulassen, jedoch infolge der dafür notwendigen Kooperation mit den Verwaltungsadressaten Interessenkonflikte in Kauf genommen werden (S.143f.). 
5 Zwischen den Beschreibungen der Begrifflichkeiten und der Darstellung der Aspekte der Erfüllungsprivatisierung befasst sich Elser mit den verfassungsrechtlichen Anforderungen an die Erfüllung staatlicher Aufgaben (§3). Er macht diese in den rechtsstaatlichen Grundsätzen von Art. 5 BV (Gesetzmässigkeit, öffentliches Interesse, Verhältnismässigkeit, Treu und Glauben) sowie der Grundrechtsbindung aus. Mit Bezug auf die Grundrechtsbindung führt Elser dabei in überzeugender Weise aus, dass ein funktioneller Ansatz zu verfolgen ist und somit eine Grundrechtsbindung nur für Tätigkeiten eines Akteurs besteht, die zum Bereich einer staatlichen Aufgabe gehören (S. 66 ff.). Demgegenüber werden allgemeine Grundsätze wie das Subsidiaritätsprinzip oder der Grundsatz der Staatsfreiheit der Wirtschaft sowie die Pflicht, Grundversorgungsleistungen zu erbringen, als verfassungsrechtliche Anforderungen an das staatliche Handeln verworfen (S. 80 ff.). In Anbetracht dieser Herausarbeitung allgemein gültiger verfassungsrechtlicher Anforderungen darf allerdings nicht vergessen werden, dass für bestimmte öffentliche Aufgaben spezifische verfassungsrechtliche Vorgaben bestehen, die auf Organisations-, Handlungs-, oder Finanzierungsformen der Erfüllung staatlicher Aufgaben durchschlagen. $\mathrm{Zu}$ denken ist beispielsweise an Art. $81 a$ Abs. 2 BV, wonach die Kosten des öffentlichen Verkehrs zu einem angemessenen Teil durch von den Nutzern bezahlten Preisen zu decken sind.

6 In den $\$ \S 5$ und 6 werden die Aspekte der Erfüllungsprivatisierung (Organisations-, Handlungs- und Finanzierungsformen) an den verfassungsrechtlichen Anforderungen (Gesetzmässigkeit, öffentliches Interesse, Verhältnismässigkeit, Treu und Glauben, Grundrechte) gemessen. In \$ 5 wird dabei dargestellt, welche Probleme durch die Einhaltung der verfassungsrechtlichen Anforderungen bei der privatisierten Aufgabenerfüllung verursacht werden. Die Dissertation bewegt sich diesbezüglich ganz bewusst auf einer abstrakten Ebene. Das Verhältnis zwischen den verfassungsrechtlichen Anforderungen und der Erfüllungsprivatisierung wird nicht mit Blick auf eine bestimmte staatliche Aufgabe, sondern in allgemeingültiger Weise untersucht. Zwar kann dadurch eine generelle Matrix der Probleme aufgezogen werden (S. 202), jedoch bleiben diese zuweilen nur schwer fassbar. Exemplarisch zeigt sich dies mit Bezug auf die Anforderung der Verhältnismässigkeit. Die abstrakte Prüfung bietet hier wenig Erkenntnisgewinn, da die Verhältnismässigkeit wesentlich von der konkreten Sachlage und den infrage stehenden Interessenkonstellationen abhängt. ${ }^{2}$ Beispielsweise lässt sich die Frage, ob unver-

2 Vgl. BENJAMIN SCHINDLER, in: Ehrenzeller/Schindler/Schweizer/ Vallender (Hrsg.), St. Galler Kommentar, Die schweizerische Bundesverfassung, 3. Aufl., Zürich 2014, Art. 50 N 50. bindliche, «privatisierte» Handlungsinstrumente wie Warnungen und Empfehlungen - wie von Elser geltend gemacht (S. 183f. und 202) - möglicherweise weniger geeignet zur Verfolgung öffentlicher Interessen sind als Verbote oder Bewilligungspflichten, kaum abstrakt beurteilen. ${ }^{3}$

Allerdings erlaubt es der von Elser gewählte Ansatz, eine 7 Übersicht der Problematiken in anschaulicher Weise aufzuzeigen, ohne sich in den Einzelheiten spezifischer Privatisierungsvorhaben zu verzetteln. Dadurch gelingt es Elser, den Finger auf mehrere wunde Punkte zu legen, die bezüglich der unterschiedlichen Aspekte der Erfüllungsprivatisierung bestehen. So sieht er beispielsweise den Grundrechtsschutz bei privatisierten Organisationsformen beeinträchtigt, da diese nur punktuell und nachträglich beaufsichtigt werden (S.193 und 234), und mahnt, dass privatisierte Finanzierungsformen in der Gestalt von Kostentragungspflichten von der Ausübung von Grundrechten abschrecken können (S.195). Als Querschnittsprobleme, die sich über die einzelnen verfassungsrechtlichen Anforderungen und Aspekte der Erfüllungsprivatisierung hinweg stellen, werden Rechtsschutzdefizite des Zivilrechts, zusätzlicher Kontroll- und Aufsichtsaufwand sowie eine erschwerte Erkennbarkeit des Staatshandelns identifiziert (S.197ff.). Letzteres bezeichnet die Gefahr, dass die Erfüllungsprivatisierung staatliches Handeln im privaten Kleid erscheinen lässt, infolgedessen Verwaltungsadressaten - in Unkenntnis darüber, dass sie es mit dem Staat zu tun haben - keinen Gebrauch von ihren verfassungsrechtlichen Ansprüchen machen.

In $\$ 6$ wendet sich Elser nun den Spielräumen zu, welche 8 die verfassungsrechtlichen Anforderungen bei der privatisierten Erfüllung staatlicher Aufgaben belassen. Denn «nur soweit solche Spielräume bestehen, [...] kann die Erfüllungsprivatisierung von Freiräumen profitieren und so zu einer insgesamt effizienteren Aufgabenerfüllung führen» und nur in diesem Fall ist die Erwartung von weniger Staat berechtigt (S. 205). Dabei werden in Bezug auf die Aspekte der Erfüllungsprivatisierung und der jeweiligen verfassungsrechtlichen Anforderungen Spielräume untersucht und anschliessend in einer übersichtlichen Matrix zusammengestellt (S. 229). Diese Zusammenstellung zeigt ein eindrückliches Bild an potenziellen Spielräumen. Mit Blick auf privatisierte Handlungsformen (wie insbe-

3 Bspw. Ist die Love-Life-Kampagne des BAG, welche eine behördliche Warnung oder Empfehlung darstellt (vgl. BGE144 II 233 E. 4.2), wohl ein sehr geeignetes Handlungsinstrument mit Bezug auf den Schutz der Bevölkerung vor HIV und anderen sexuell übertragbaren Krankheiten. Demgegenüber wurde die im Rahmen der Covid19-Pandemie abgegebene behördliche Empfehlung, eine Maske im öffentlichen Verkehr zu tragen, ungenügend befolgt, weshalb eine Maskenpflicht eingeführt werden musste. 
sondere konsensuale Instrumente) wird etwa aufgezeigt, dass geringere Anforderungen an die Normdichte der gesetzlichen Grundlage bestehen (S. 211) und solche einvernehmlichen Handlungsformen infolge der Einbindung privater Interessen eher zur Zumutbarkeit staatlicher Eingriffe führen (S. 218f.). Etwas offen bleibt in diesem Abschnitt, zu welchem Zweck die Spielräume denn genutzt werden können. In dieser Hinsicht rächt es sich, dass sich die Dissertation nicht vertieft mit den Motiven für die Erfüllungsprivatisierung auseinandersetzt, hätte dies doch eine schärfere Ausarbeitung der Spielräume erlaubt.

9 In $\$ 7$ erfolgt eine prägnante Übersicht der Untersuchungsergebnisse. Elser belässt es allerdings nicht dabei, sondern erstellt aus den herausgearbeiteten Problemfeldern Checklisten, die mit Bezug auf beabsichtigte Privatisierungen der Organisations-, Handlungs-, und Finanzierungformen durchzugehen sind (S. 237 f.). Diese Checklisten umfassen durchwegs relevante Fragen, mit welchen die vom Autor aufgezeigten potenziellen verfassungsrechtlichen Problematiken bei Privatisierungen nochmals greifbar gemacht werden. Die Dissertation schliesst mit verschiedenen Empfehlungen (S. 239f.). Elser weist etwa auf die Notwendigkeit hin, gesetzlich möglichst präzise festzulegen, welche Aufgaben einem Akteur zugewiesen werden. Ebenfalls rät er davon ab, bei einem Aufgabenträger die Erfüllung öffentlicher Aufgaben mit privatwirtschaftlichen Tätigkeiten zu verbinden. Angesichts der zahlreichen hybrid tätigen öffentlichen Unternehmen (insbesondere der Energieversorgungsunternehmen), bestehen gewisse Zweifel, ob diese grundsätzlich berechtigte Forderung in der Praxis auf offene Ohren stossen wird.

\section{Würdigung}

Elser geht es in seiner Dissertation nicht darum, sämtli- 10 che Fragen, die sich in Bezug auf die unterschiedlichen Privatisierungsformen der zahlreichen öffentlichen Aufgaben stellen, zu beantworten. Vielmehr nimmt er aus einer Vogelperspektive eine Analyse vor, wie die privatisierte Erfüllung öffentlicher Aufgaben im Lichte der verfassungsrechtlichen Anforderungen einzuordnen ist. Hervorzuheben ist dabei, wie es dem Autor gelingt, die Perspektiven der Organisations-, Handlungs-, und Finanzierungsformens umfassend in das Themenfeld der «Privatisierung» einzubinden. Dadurch öffnet Elser die Privatisierungsdiskussion und bereitet ein weites Feld für zukünftige Untersuchungen vor. So könnte anhand der Erfüllungsprivatisierung einer bestimmten staatlichen Aufgabe (z.B. regionaler Personenverkehr) untersucht werden, inwiefern und mit welchen Mitteln den Problemen der Erfüllungsprivatisierung Rechnung getragen wurde und inwieweit die verfassungsrechtlichen Spielräume genutzt wurden.

Insgesamt ist Elser mit seiner Dissertation ein frischer 11 Blick auf eine bedeutende Thematik gelungen. Die Dissertation vermeidet langwierige Exkurse und unnötige Darstellungen von bekannten Allgemeinschauplätzen. Vielmehr geht Elser in den sieben Abschnitten seiner Dissertation sehr zielgerichtet vor und versteht es, den Leser gekonnt durch seine klar strukturierten Überlegungen zu führen. Die Arbeit ist damit äusserst übersichtlich und liest sich wie «aus einem Guss», wodurch sie bei zukünftigen Privatisierungsvorhaben staatlicher Aufgaben hoffentlich ihre berechtigte Berücksichtigung finden wird.

Rezensiertes Werk:

«Die privatisierte Erfüllung staatlicher Aufgaben», Dominik Elser, sui generis Verlag, Zürich 2020

ISBN: 978-3-907297-09-4 (gedruckte Ausgabe, Hardcover)

DOI: $10.38107 / 009$ (e-Book, Open Access) 